\title{
New Yb:Hf-doped silica fiber for high-power fiber lasers
}

\author{
J.K.Sahu, P.Dupriez, J.Kim, A.J.Boyland. C.A.Codemard, J.Nilsson, and D.N.Payne \\ Optoelectronics Research Centre, University of Southampton
}

\begin{abstract}
We report an efficient cladding-pumped $\mathrm{Yb}$-doped $\mathrm{HfO}_{2}-\mathrm{SiO}_{2}$ fiber laser that generated $122 \mathrm{~W}$ of continuous-wave output power at $1.08 \mu \mathrm{m}$ with a slope efficiency of $74 \%$ with respect to the absorbed pump power.
\end{abstract}

\section{Introduction}

The remarkable advances in fiber laser output powers in recent years [1] have led to the search for novel dopants in silica-based fiber with improved glass properties, such as good control of refractive index, better rare-earth (RE) solubility and high optical damage threshold. To date, almost all efficient high-power fiber lasers have been realized in $\mathrm{Al}_{2} \mathrm{O}_{3}-\mathrm{SiO}_{2}$ hosts. This is due to the fact that $\mathrm{Al}_{2} \mathrm{O}_{3}$ allows a higher $\mathrm{RE}$ doping level into the silica matrix without clustering effects than that possible either in pure, or in germaniumbased, silica hosts. $\mathrm{Al}_{2} \mathrm{O}_{3}$ also broadens the emission spectrum of RE ions in a silica host. Unfortunately, an $\mathrm{Al}_{2} \mathrm{O}_{3}$ content exceeding 4 mol\% leads to devitrification [2]. This makes it difficult to achieve a high numerical aperture (NA) through $\mathrm{Al}_{2} \mathrm{O}_{3}$ co-doping. On the other hand, high-power lasers require large core, low NA fiber design to limit the core optical intensity, which implies a low concentration of alumina codopant. Unfortunately, this leads to low RE concentrations because of the problem of clustering in the low alumina regime. High RE concentrations are required to shorten the device length of high-power fiber lasers, which helps to suppress nonlinear effects, such as stimulated Raman and Brillouin scattering. In this work we investigate the use of hafnium as an alternative to aluminum in a RE-doped silica matrix. Hafnia, $\mathrm{HfO}_{2}$, is a high refractive index material and is transparent over a wide wavelength range $(0.3-10 \mu \mathrm{m})$. Hafnia and crystalline silicon dioxide layers have been used in combination to form high index-contrast multilayer structures with high laser damage threshold [3]. Thus, RE-doped $\mathrm{HfO}_{2}-\mathrm{SiO}_{2}$ fiber laser systems look promising for high-power, high-energy short-pulse generation that requires a core material with high optical damage threshold.

Here, we present initial results in what we believe to be the first report of a high-power cladding pumped ytterbium-doped hafnia-silica (Hf:Si) fiber laser. The laser generated $122 \mathrm{~W}$ of continuous-wave (CW) output power at $1080 \mathrm{~nm}$ with a slope efficiency of $74 \%$, with respect to absorbed pump power, in a relatively high brightness beam, $\mathrm{M}^{2}=2.8$. The output power was limited by available pump power. This is the first new co-dopant reported for silica fibers for many years.

\section{Experiments and results}

The double-clad Yb:Hf-doped fiber used in this work was fabricated in-house by modified chemical vapor deposition (MCVD) combined with the solution-doping technique [4]. Silica soot was first deposited on the inside of a substrate tube, and the soot was then immersed in a $\mathrm{HfCl}_{4}$ and $\mathrm{YbCl}_{3}$ solution. The soot was dried in a flow of dry $\mathrm{O}_{2}$ and $\mathrm{N}_{2}$ gases and then consolidated to form a glass layer. The preform tube was subsequently collapsed into a solid preform. Before being drawn into fiber, the preform was milled to a Dshape in order to improve the pump absorption in the doped core. This preform was drawn to a $30 \mu \mathrm{m}$ core (NA 0.1) fiber with a 400/360 $\mu \mathrm{m}$ pure silica inner cladding diameter (long/short axis dimensions) and coated with a low-index polymer outer cladding, which provided a nominal NA of $\sim 0.48$. The outer diameter was chosen to enable efficient pump launch from the high-power pump source used in this work. The small signal cladding absorption at the pump wavelength of $975 \mathrm{~nm}$ was $\sim 1 \mathrm{~dB} / \mathrm{m}$. The core background loss, measured with a white light source in a $125 \mu \mathrm{m}$ OD fiber drawn from the same preform, was found to be $\sim 12 \mathrm{~dB} / \mathrm{km}$ at $1200 \mathrm{~nm}$. 
The laser configuration used in our experiment is shown in Figure 1 . A $10 \mathrm{~m}$ long piece of the large-core Yb:Hf-doped fiber is pumped by a $975 \mathrm{~nm}$ diode-stack laser through a combination of collimating and focusing lenses. We could launch as much as $80 \%$ of the pump power into the fiber. The laser cavity is formed between a perpendicularly cleaved fiber facet at the pump launch end and a highly reflecting external mirror at the other end of the fiber. A dichroic mirror with high reflection at $1-1.1 \mu \mathrm{m}$ and high transmission at the $975 \mathrm{~nm}$ pump wavelength was used to separate the signal output from the pump beam at the pump launch end of the fiber.

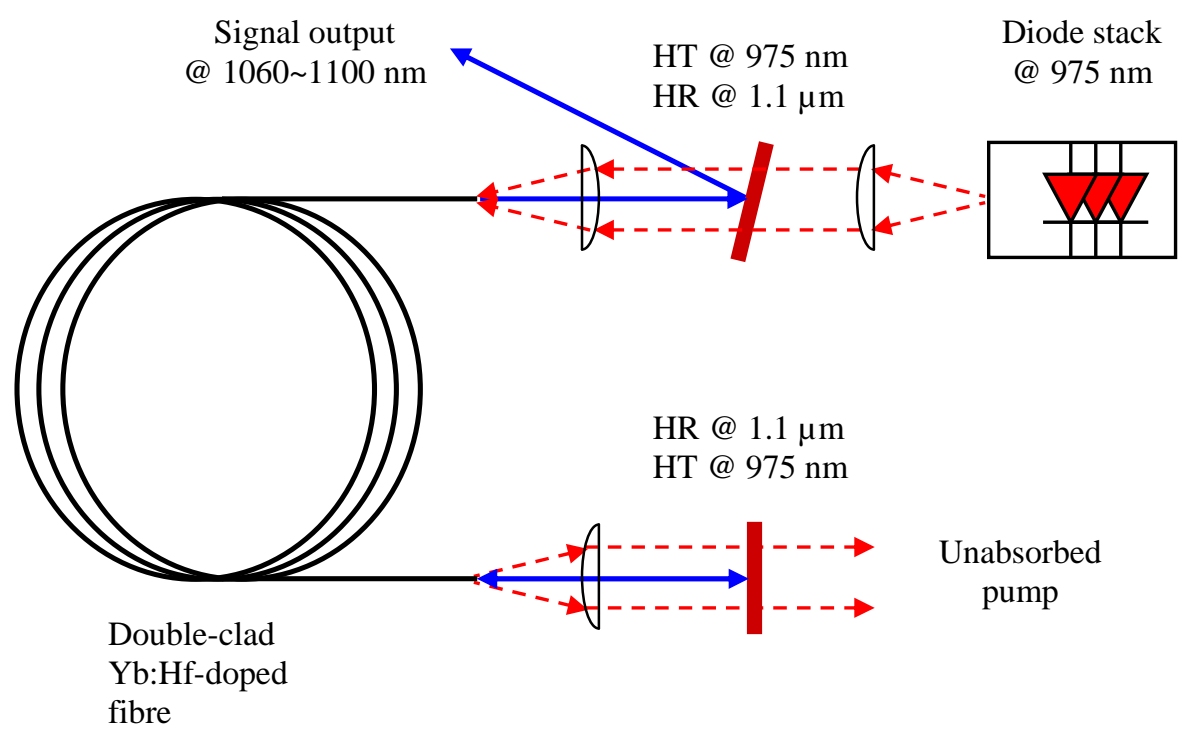

Figure 1 . Experimental set-up of cladding-pumped Yb-doped fiber laser. HT: High transmission. HR: high reflection.

Figure 2 shows the laser output characteristics, together with the output spectrum measured at maximum output power. The output power reached $122 \mathrm{~W}$ for an absorbed pump power of $165 \mathrm{~W}$. The slope efficiency was $74 \%$ with respect to the absorbed pump power, which is comparable with an $\mathrm{Al}_{2} \mathrm{O}_{3}$ host. The laser emission was centered at $\sim 1080 \mathrm{~nm}$ and the spectrum was similar to that obtained in Ref. [1]. The output power increased linearly with the pump power, and no evidence of power roll-off was observed even at the highest pump power. The measured beam quality factor $\left(\mathrm{M}^{2}\right)$ was 2.8 . It is worth mentioning that no special care has been taken to suppress the higher order transverse modes in order to improve the beam quality. The standard deviation of the short-term stability of the output power was below $3 \%$, measured with a $5 \mathrm{GHz}$ photo-detector and a $400 \mathrm{MHz}$ bandwidth oscilloscope. The measured lifetime of the Yb-ions was $775 \mu$ s compared to $800 \mu$ s in a similar aluminosilicate host core perform.

\section{Conclusion}

We have demonstrated cw operation of an efficient double-clad Yb:Hf -doped large core silica fiber laser, and obtained an output power of $122 \mathrm{~W}$ at $1080 \mathrm{~nm}$ with a beam quality ( $\mathrm{M}^{2}$-value) of 2.8. The slope efficiency was $74 \%$ with respect to the absorbed pump power and no roll over was observed in output power with pump power. We are currently working on further power scaling with improved beam quality in this fiber. These promising first results suggest that hafnium is an interesting alternative co-dopant to the commonly-used aluminum in RE-doped silica fiber cores for high power fiber lasers and amplifiers. On going work to demonstrate the limits of RE solubility in hafnia co-doped fibers will be presented with a view to a full evaluation of the merits of hafnia. 

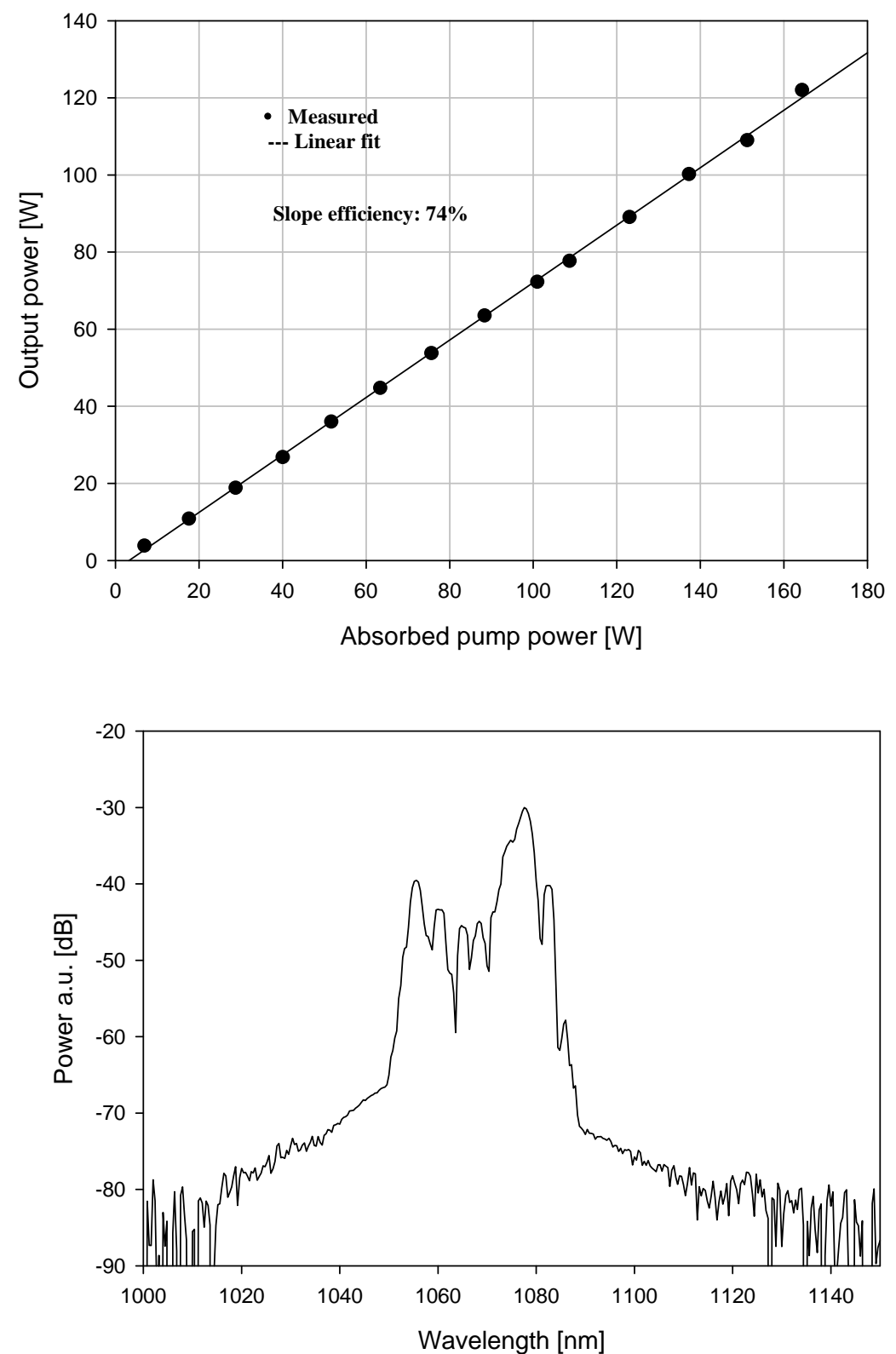

Figure 2. Laser output power versus launched pump power (upper) and output spectrum at maximum output power (lower).

\section{References}

1. Y. Jeong, J. K. Sahu, D. N. Payne, J. Nilsson, "Ytterbium-doped large-core fibre laser with $1 \mathrm{~kW}$ continuous-wave output power”, Electron. Lett. 40, 470-472 (2004).

2. C. J. Scott, "Optimization of composition for $\mathrm{Al}_{2} \mathrm{O}_{3} / \mathrm{P}_{2} \mathrm{O} 5$-doped optical fiber", Proc. Conf. on Optical Fiber Communication, New Orleans, USA, Jan 23-35, 1984, paper TUM4.

3. Technical information, CERAC, Inc (http://www.cerac.com/pubs/proddata/hfo2.htm).

4. J. E. Townsend, S. B. Poole, and D. N. Payne, "Solution doping technique for fabrication of rareearth doped optical fibres", Electron. Lett. 23, 329-331 (1987). 